\title{
Electric Double-Layer Capacitor Performance of a New Mesoporous Carbon
}

\author{
Songhun Yoon, Jinwoo Lee, Taeghwan Hyeon, and Seung M. Oh*,z \\ School of Chemical Engineering and Institute of Chemical Processes, Seoul National University, Seoul 151-742, South Korea
}

\begin{abstract}
A new mesoporous carbon (NMC) was prepared, and its performance in an electric double-layer capacitor (EDLC) was compared to that of a conventional carbon (a molecular-sieving carbon, MSC25). The effect of pore size and pore connection pattern on EDLC performance was demonstrated. To prepare NMC, phenol resin was synthesized inside the pores of an inorganic template, Mobile Composite Material 48 (MCM48), and the resulting resin-template composite was carbonized at $700^{\circ} \mathrm{C}$ under $\mathrm{Ar}$ atmosphere. A coke-like carbonaceous material was obtained after removing the inorganic template by HF treatment. The surface area of NMC was $1257 \mathrm{~m}^{2} \mathrm{~g}^{-1}$ which is smaller than that of MSC25 $\left(1970 \mathrm{~m}^{2} \mathrm{~g}^{-1}\right)$. NMC had three-dimensionally interconnected mesopores $(2.3 \mathrm{~nm}$ average diam), but randomly connected cage-like micropores $(<2.0 \mathrm{~nm})$ were dominant in MSC25. The difference in the pore size and pore connection pattern between the two carbons gave rise to a remarkable difference in their EDLC performances. NMC exhibited a smaller specific capacitance (about $120 \mathrm{~F} \mathrm{~g}^{-1}$ ) than MSC25 as a result of its smaller surface area, but it showed a higher critical scan rate than the MSC25 electrode due to a smaller resistance-capacitance (RC) time constant. The specific charging capacity of the NMC electrode was about $20 \mathrm{mAh} \mathrm{g}^{-1}$ and was largely invariant $v s$. the charge-discharge rate. This was contrasted by MSC 25 which showed a steadily decreasing capacity with an increase in rate. As a result, the NMC electrode outperformed the MSC25 based on rate capability. The smaller $R C$ time constant and better rate capability of the NMC electrode apparently arises from the lower electrolyte resistance in pores, which in turn stems from the faster ionic motion in larger pores.

(c) 2000 The Electrochemical Society. S0013-4651(00)01-080-6. All rights reserved.
\end{abstract}

Manuscript submitted January 20, 2000; revised manuscript received April 3, 2000.

The electric double-layer capacitor (EDLC) has been considered as a promising high power energy source for digital communication devices and electric vehicles. The advantageous features of the EDLC are its better rate capability and longer cycle life as compared to modern secondary batteries. EDLC utilizes the double layer formed at electrode/electrolyte interface where electric charges are accumulated on the electrode surfaces and ions of opposite charge are arranged in the electrolyte side. EDLC electrode materials should thus have a large surface area for charge accumulation, and should have an appropriate pore structure for electrolyte wetting and rapid ionic motion. At present, activated carbons or molecular-sieving carbons are used as the EDLC electrode materials. Even if these conventional carbons have a large surface area, their EDLC application is rather limited because they contain pores ranging from micropores $(<2 \mathrm{~nm}$ diam) to macropores and the pores are randomly connected. ${ }^{1}$ The micropores are not easily wetted by electrolytes, and the exposed surface in micropores may not be utilized for charge storage. Moreover, even in the situation wherein the micropores are wetted by electrolyte, ionic motion in such small pores may be so slow that the high rate capability, which is one of the advantages of EDLCs, may not be realized. ${ }^{1}$ Both charge storage and rate capability are further limited if the pores are randomly connected. The blind or isolated pores may not be wetted by electrolytes and irregular pore connection makes ionic motion difficult. ${ }^{2,3}$ Therefore, high-surface-area carbon materials containing regularly interconnected mesopores ( $>2 \mathrm{~nm}$ ) are highly desirable for the EDLC electrode.

Recently, we have synthesized a new mesoporous carbon (NMC) that appears to generally meet the above requirements. It was prepared via the template route, in which the mesoporous aluminosilicates were utilized as the template., 4 Phenol resin was prepared inside the pores of the template and carbonized. Mesoporous carbon with three-dimensionally interconnected $c a .2 \mathrm{~nm}$ pores was obtained after removing the inorganic template with a hydrofluoric acid treatment.

As an extension of our previous report, this paper deals with the physicochemical properties of NMC and its EDLC performance. The pore structure and electrical conductivity of NMC were measured, and its EDLC performance characteristics including the capacitance and rate capability were analyzed. Similar measurements were car-

\footnotetext{
* Electrochemical Society Active Member.
}

z E-mail: seungoh@plaza.snu.ac.kr ried out with a molecular-sieving carbon (MSC25) that has random cage-like micropores $(<2 \mathrm{~nm})$, and the effect of pore size and pore connection pattern on EDLC performances of carbon materials was discussed.

\section{Experimental}

Materials. - The synthetic procedure for NMC was provided in our preliminary report. ${ }^{4}$ For the synthesis, a mesoporous aluminosilicate identified as Mobile Composite Material 48 (MCM48) was used as the template. The molecular-sieving carbon (MSC25) provided by Kansai Coke and Chemicals has a specific Brunauer, Emmett, and Teller (BET) surface area of $1970 \mathrm{~m}^{2} \mathrm{~g}^{-1} .6$

To analyze the pore and crystal structure of both carbons, smalland wide-angle X-ray diffraction (XRD) patterns were obtained with a Rigaku D/Max-3C diffractometer with a rotating anode and $\mathrm{Cu} \mathrm{K} \alpha$ radiation $(\lambda=0.15418 \mathrm{~nm})$. The BET surface area and pore size distribution was calculated from $\mathrm{N}_{2}$ adsorption isotherms using the Barrett, Joyner, and Halenda $(\mathrm{BJH})$ method. The isotherms were collected at $77 \mathrm{~K}$ with a gas adsorption analyzer (Micrometrics ASAP2010) after the carbon materials were degassed at $250^{\circ} \mathrm{C}$ for $5 \mathrm{~h}$ under $30 \mu$ Torr. The four-probe van der Pauw method was utilized to measure the electrical conductivity of carbon powders. ${ }^{7}$ For this measurement, carbon powder was placed in a polyethylene cylinder with one end closed. The conductivity was measured with the four probes contacted to the powder sample while the pressure was adjusted by a polyethylene rod. ${ }^{8}$

To prepare the NMC-containing composite electrodes, a mixture of NMC and polytetrafluoroethylene (PTFE) binder (10:1 weight ratio) was dispersed in isopropyl alcohol and coated on a stainless steel Exmet $(1 \times 1 \mathrm{~cm})$ which was used as the current collector. The resulting electrode plate was pressed and dried under vacuum at $120^{\circ} \mathrm{C}$ for $12 \mathrm{~h}$. For the preparation of the MSC25 composite electrode, however, an excess amount of Ketjenblack ECP-600JD (KB) was added to increase the electrode conductivity. Each composite electrode contained about $10 \mathrm{mg}$ of carbon material (NMC or MSC25). Electrical conductivity of the composite carbon electrodes was measured with the common van der Pauw method.

Electrochemical characterizations.-The electrochemical performance of the composite carbon electrodes was analyzed with a threeelectrode configuration. The electrolyte was aqueous $2.0 \mathrm{M} \mathrm{H}_{2} \mathrm{SO}_{4}$ unless otherwise specified. A Pt flag and saturated calomel electrode 
(SCE) were used as the counter and reference electrode, respectively. Cyclic voltammetry was performed using an EG\&G PARC 362 potentiostat in the potential range of 0.0 to $0.7 \mathrm{~V}$ ( $v s$. SCE) with a scan rate from 5 to $50 \mathrm{mV} \mathrm{s}^{-1}$. The current in the resulting cyclic voltammograms was divided by the scan rate to obtain capacitance vs. voltage profiles. Galvanostatic charge-discharge voltage profiles were obtained using a current density from 0.1 to $60 \mathrm{~mA} \mathrm{~cm}^{-2}$, from which the specific capacitance of the carbon materials was estimated.

Chronoamperometry was utilized to estimate the $R C$ time constant $[\tau, R$ is the equivalent series resistance (ESR) and $C$ is the capacitance] of the electrochemical system. ${ }^{9,10}$ For this measurement, a potential step $(\Delta E=10 \mathrm{mV})$ was applied from $0.2 \mathrm{~V}$ by combining an EG\&G PARC 362 potentiostat and programmable voltage source, and the resulting current transients were fitted with $I(t)=(\Delta E / R) \exp (-\mathrm{t} / \tau)$ to extract the ESR, $C$, and $\tau$ values. With an assumption that the ESR is composed of the electrode resistance $\left(R_{\text {electrode }}\right)$, bulk electrolyte resistance $\left(R_{\text {bulk }}\right)$, and electrolyte resistance in pores $\left(R_{\text {pore }}\right)$, the $R_{\text {pore }}$ was calculated from the measured values of the former parameters.

\section{Results and Discussion}

Characterization of materials.-Figure 1a shows the small-angle XRD patterns of two carbon materials. As seen, NMC exhibits intense peaks at $2 \theta=1.6$ and $2.7^{\circ}$ that arise from the long-range ordering of pores. ${ }^{4}$ In contrast, MSC 25 shows no peaks in this range, indicating that the pores are randomly distributed. The literature also suggested that randomly connected cage-like micropores $(<2 \mathrm{~nm})$
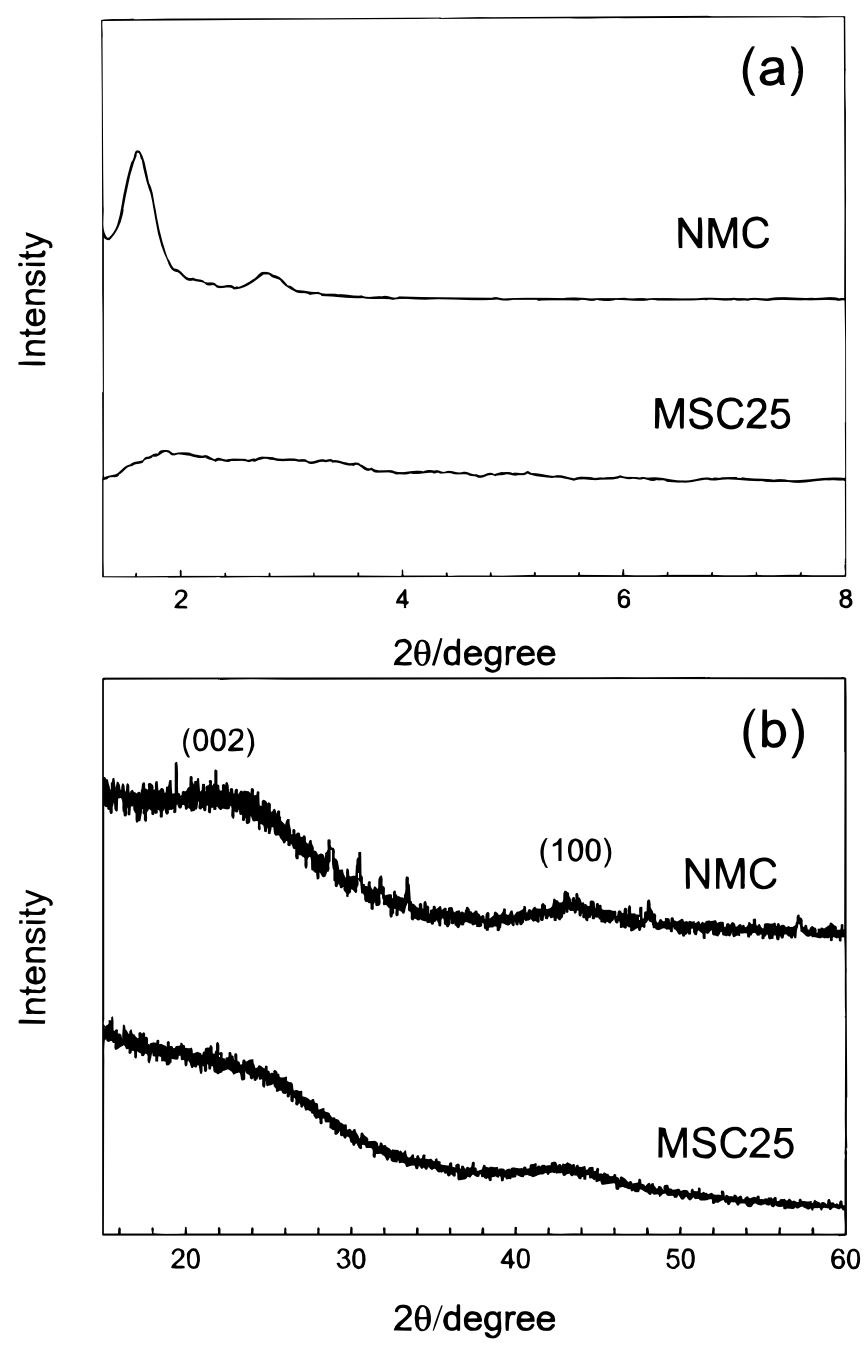

Figure 1. (a) Small-angle XRD patterns of new mesoporous carbon (NMC) and molecular-sieving carbon (MSC25), and (b) wide-angle XRD patterns. are dominant in this carbon. ${ }^{6}$ Figure $1 \mathrm{~b}$ displays the wide-angle XRD patterns of two carbons. Both carbons show broad diffraction peaks at $2 \theta=24$ and $43^{\circ}$ that are related to the (002) and (100) reflections, respectively. ${ }^{11}$ The location and broadness of two diffraction peaks illustrate that both carbons have a coke-like character with disordered carbonaceous interlayers. ${ }^{11}$ Note that the present carbonization temperature is far below the normal graphitization temperature. Elemental analysis revealed a $\mathrm{C} / \mathrm{H}$ ratio of 9.3 and $<1 \mathrm{~mol} \%$ aluminosilicate residue in NMC $(93.24 \% \mathrm{C}, 84 \% \mathrm{H}$, $0.09 \% \mathrm{~N}$, and $2.56 \% \mathrm{O}$ ). The specific surface area of NMC was found to be $1257 \mathrm{~m}^{2} \mathrm{~g}^{-1}$ from the nitrogen BET adsorption measurement. This is smaller than that of MSC25 $\left(1970 \mathrm{~m}^{2} \mathrm{~g}^{-1}\right)$. Figure 2 shows the pore size distribution of two carbons, indicating that NMC has mesopores of $2.3 \mathrm{~nm}$ in average pore diameter, whereas micropores of $<2 \mathrm{~nm}$ are dominant in MSC25.

Figure 3 shows the electrical conductivity of two carbons. NMC exhibits a higher conductivity than MSC25. At present, no tenable explanation can be given for this observation, but note that $\mathrm{Wu}$ and Bein ${ }^{12}$ reported that a carbon/mesoporous silica composite, similarly prepared by the template route, exhibited a remarkably high conductivity. These authors ascribed this observation to the formation of conducting carbon nanowires inside mesopores. If this is the case in NMC, as NMC has long-range ordering of its carbon network, threedimensionally connected nanosized conducting wires may facilitate electrical conduction. In general, EDLC electrodes are formulated into a plate by adding polymeric binder. Therefore, the electrode conductivity, rather than the electrical conductivity of the carbon power itself, is the practically important variable affecting EDLC performance. Table I lists the conductivity of composite EDLC electrodes measured by the van der Pauw four-probe method. The NMCpolytetrafluoroethylene (PTFE) composite electrode exhibits a higher conductivity $\left(0.59 \mathrm{~S} \mathrm{~cm}^{-1}\right)$ than the MSC25-PTFE electrode $\left(0.10 \mathrm{~S} \mathrm{~cm}^{-1}\right)$, which is certainly the result of the conductivity difference between two carbon materials. The conductivity of the MSC25-PTFE electrode can, however, be raised by adding a con-

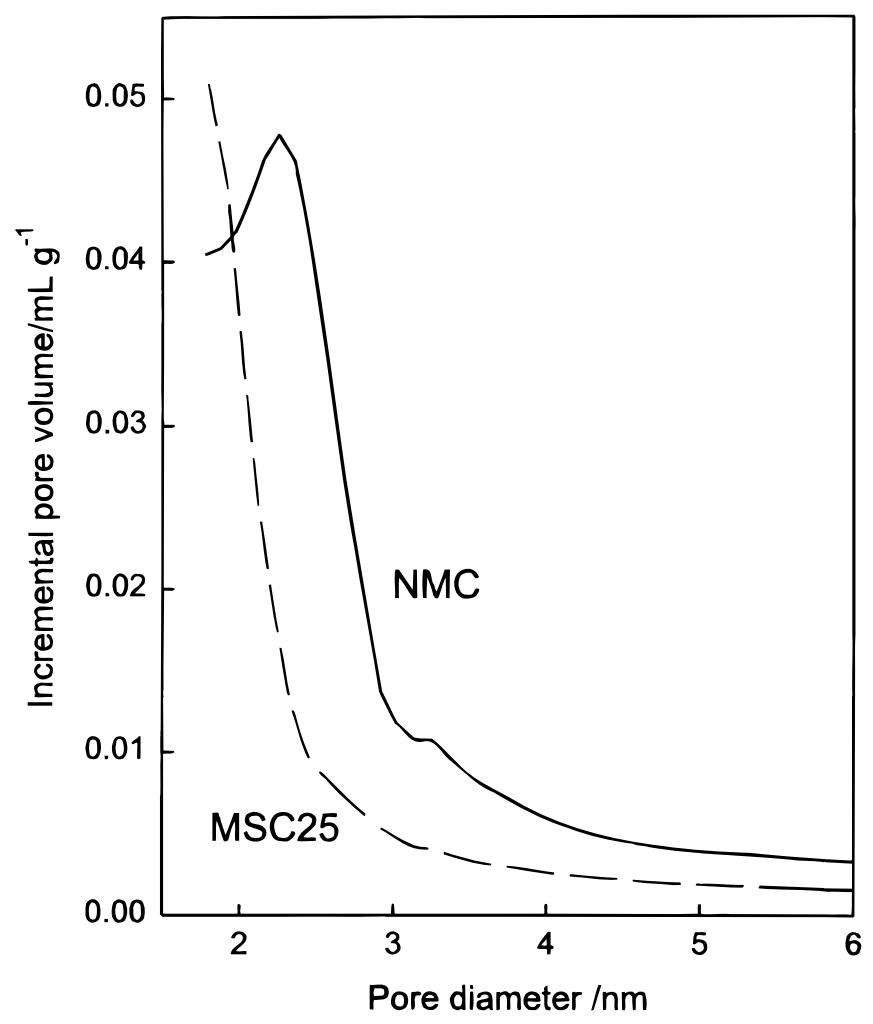

Figure 2. Pore size distribution of two carbons that was measured by $\mathrm{N}_{2}$ adsorption isotherms. 


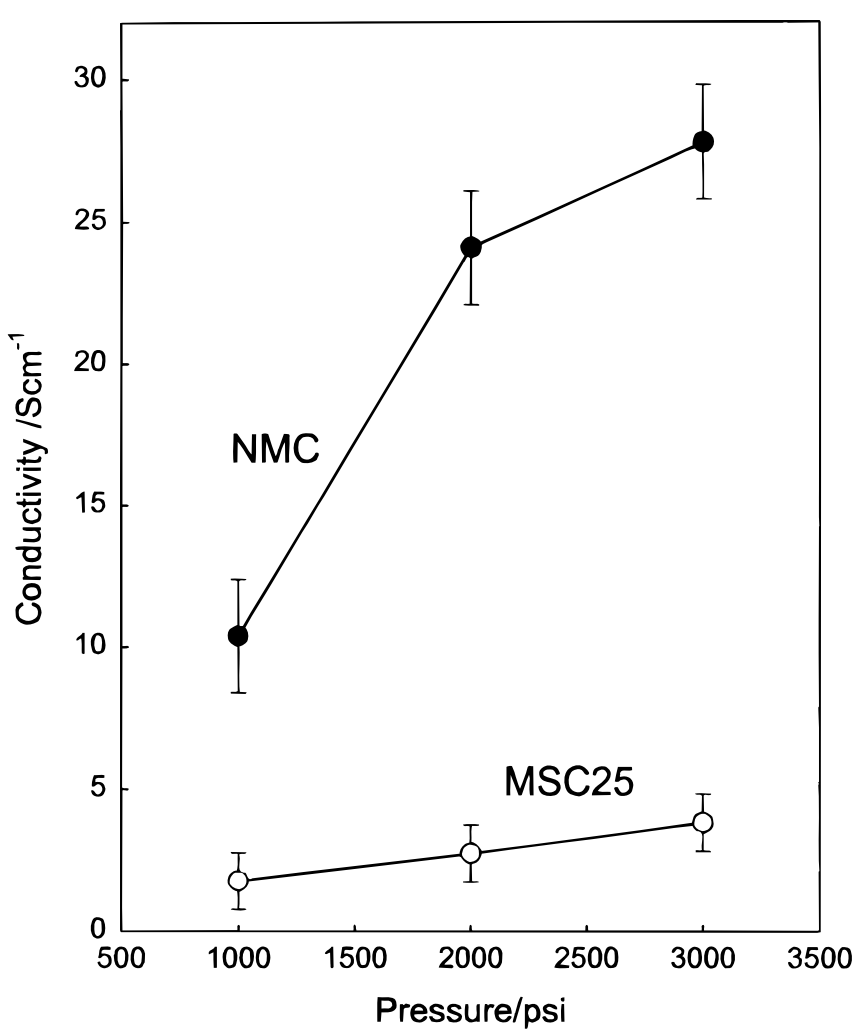

Figure 3. Change of dc conductivity with applied pressure. The dc conductivity measurement was carried out using the modified van der Pauw method. Experimental details are described in the text.

ducting aid. As shown, when 7\% of Ketjenblack is added, its conductivity becomes comparable to that of the NMC-PTFE electrode.

EDLC performance of two carbon electrodes.-Figure 4 displays cyclic voltammograms obtained for three carbon composite electrodes. In general, the equivalent circuit for EDLC electrodes can be represented as a serial combination of equivalent series resistance (ESR) and double-layer capacitance. ${ }^{10}$ When cyclic voltammgrams are recorded with EDLC electrodes, the voltammogram shape is affected by the $R C$ time constant $(\tau)$ of the electrode system. ${ }^{10,13,14}$ If $\tau=0$, a scan rate independent rectangular-shaped profile is expected as the current decays within an infinitesimally short period after voltage switching. However, if $\tau \neq 0$, the current contains a transient part, which falls exponentially with a $R C$ time constant, and a steady-state current $(\nu C$, where $\nu$ is the scan rate and $C$ is the double-layer capacitance). ${ }^{13,14}$ Also, as $\tau$ becomes larger, the transient part lasts longer (more time is required to charge the capacitor), resulting in a collapse of the rectangular current profiles. This phenomenon certainly becomes more significant at higher scan rates. Based on this discussion and from the result provided in Fig. 4, it is

Table I. The dc conductivity of composite electrodes.

Composite electrode $\mathrm{a}^{\mathrm{a}}$

Conductivity $\left(\mathrm{S} \mathrm{cm}^{-1}\right)$

\begin{tabular}{ll}
\hline NMC & $0.59 \pm 0.01$ \\
MSC25 & $0.10 \pm 0.01$ \\
MSC25-5\% KB & $0.35 \pm 0.01$ \\
MSC25-7\% & $0.60 \pm 0.01$ \\
MSC25-10\%KB & $1.14 \pm 0.01$
\end{tabular}

${ }^{\text {a }}$ The electrodes were composed of carbon and PTFE binder (10:1 wt ratio).

b The excess amount of Ketjenblack ECP600JD (KB) was added as a conducting aid. very likely that the NMC electrode has the smallest $R C$ time constant, and the MSC25 electrode the largest value. As seen, when the voltammograms of the three electrodes recorded at the same scan rate are compared, the NMC electrode exhibits the most rectangularshaped profile.

Figure 5 shows capacitance $v s$. voltage profiles that were derived by dividing the current in the cyclic voltammograms (Fig. 4) by the scan rate. In general, if the $R C$ time constant is small such that the current reaches a steady value within the experimental time scale, a plateau is expected in the capacitance vs. voltage profiles. In the other extreme, however, a persistent current transient gives a sloping curve. The results shown in Fig. 5 are well matched to this expectation. The NMC electrode having the smallest $\tau$ gives a capacitance plateau that persists at the higher scan rates. The disappearance of plateau with an increase of scan rate is most significant in the MSC25 electrode. This observation illustrates that the time constant increases in the order of NMC $<$ MSC $25-7 \% \mathrm{~KB}<\mathrm{MSC}$. The results shown in Fig. 4 and 5 require further comments. First, when the capacitance value that was estimated from the plateau region in Fig. 5 is compared between the electrodes, the MSC25 and MSC25$7 \% \mathrm{~KB}$ electrodes deliver double the capacitance $\left(1.1 \mathrm{~F} \mathrm{~cm}^{-2}\right)$ of the NMC electrode $\left(0.6 \mathrm{~F} \mathrm{~cm}^{-2}\right)$, which may be explained by the larger surface area of the former. Second, a pseudocapacitance appears at 0.3 to $0.4 \mathrm{~V}$ ( $v s$. SCE), which is related to the charge-transfer reaction at the surface quinone/hydroquinone groups. ${ }^{15}$ Finally, an extra cathodic current appears near $0 \mathrm{~V}$ which appears to result from proton reduction. ${ }^{16}$

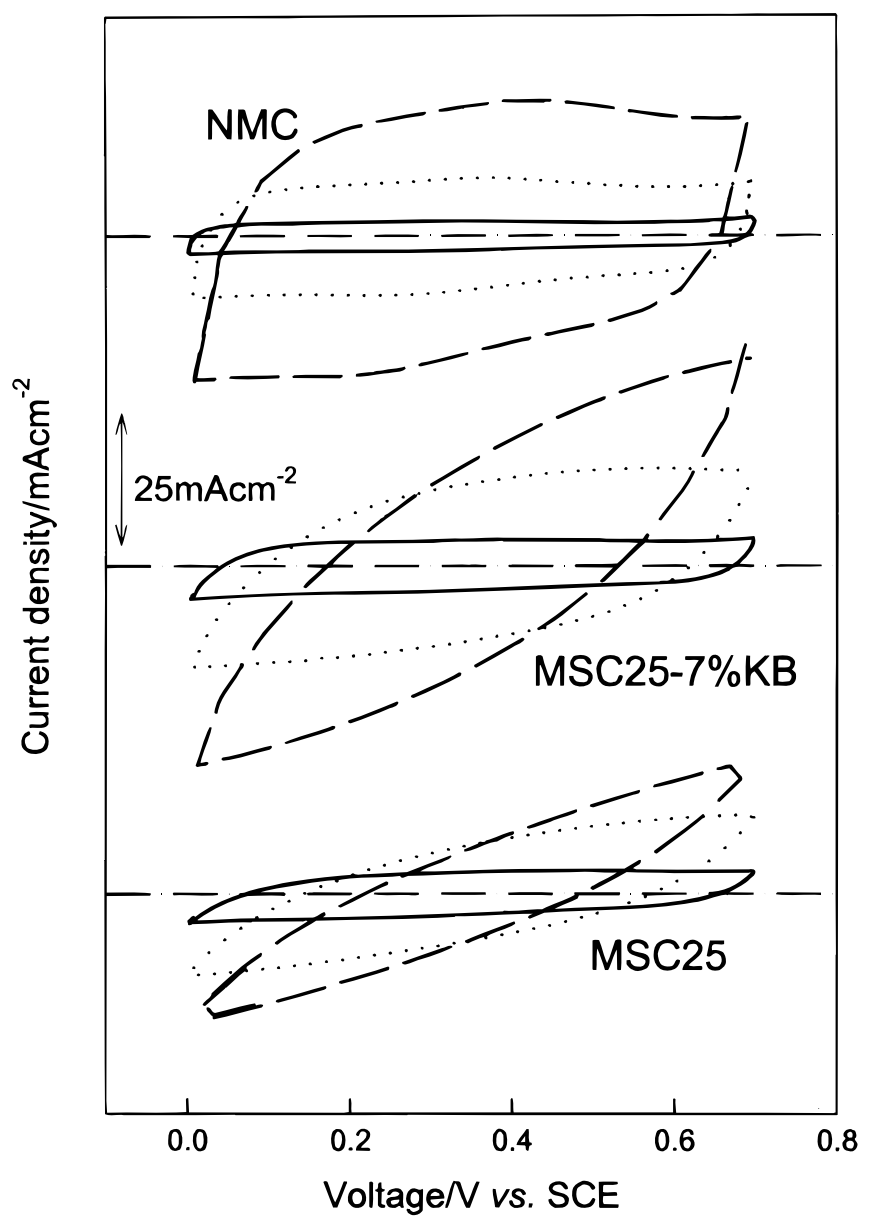

Figure 4. Cyclic voltammograms traced with three carbon composite electrodes as a function of scan rate. Scan rate $=5 \mathrm{mV} \mathrm{s}^{-1}$ (solid line), $20 \mathrm{mV}$ $\mathrm{s}^{-1}$ (dotted line), and $50 \mathrm{mV} \mathrm{s}^{-1}$ (dashed line). Note the difference in the collapse rate of the rectangular-shaped profiles as a function of scan rate. The electrolyte was aqueous $2.0 \mathrm{M} \mathrm{H}_{2} \mathrm{SO}_{4}$ solution. 


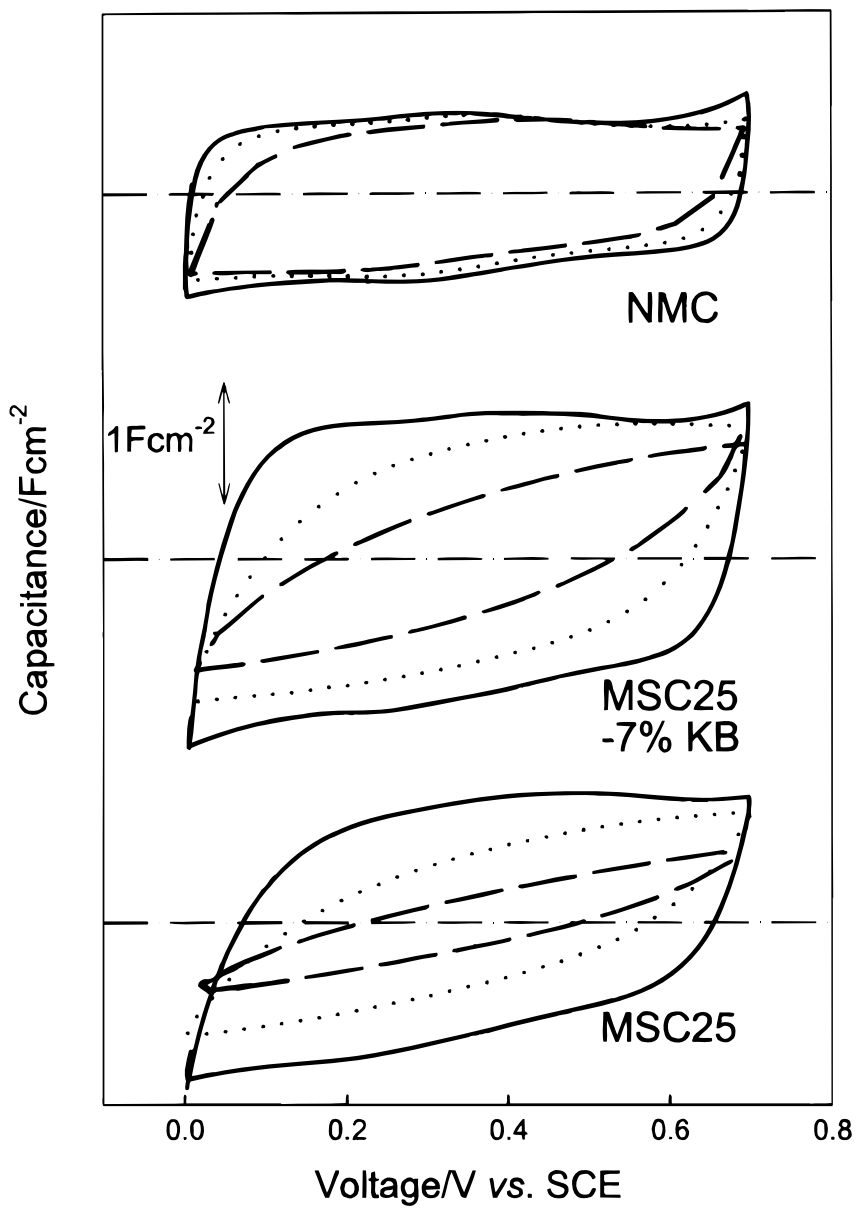

Figure 5. Capacitance $v s$. voltage profiles obtained with three carbon composite electrodes. The capacitance was calculated by dividing the current in cyclic voltammograms (Fig. 4) by the scan rate. Scan rate $=5 \mathrm{mV} \mathrm{s}^{-1}$ (solid line), $20 \mathrm{mV} \mathrm{s}^{-1}$ (dotted line), and $50 \mathrm{mV} \mathrm{s}^{-1}$ (dashed line).

Figure 6 represents the galvanostatic charge-discharge voltage profiles of the NMC and MSC25-7\%KB electrodes. Potential cycling was carried out at current densities of 5 and $50 \mathrm{~mA} \mathrm{~cm}^{-2}$ in the potential range 0.7 to $0.0 \mathrm{~V}$ ( $v s$. SCE). In the figure, the downward profiles correspond to charging (charge storage) and the upward ones to discharging (charge removal from the electrodes). When the current density increases from 5 to $50 \mathrm{~mA} \mathrm{~cm}{ }^{-2}$, the charge-discharge capacity decreases slightly in the NMC electrode whereas a significant decrease is observed with MSC25-7\% KB. The specific charging capacity of the two carbon electrodes obtained as a function of current density from 5 to $60 \mathrm{~mA} \mathrm{~cm}^{-2}$ is presented in Fig. 7. As seen, the charging capacity of the NMC electrode is about $20 \mathrm{mAh} \mathrm{g}{ }^{-1}$, which is largely invariant $v s$. current density. The MSC25-7\% KB electrode stores twice as much charge compared to the NMC electrode at the lower current density, but the values become smaller at higher current density. Therefore, the NMC electrode stores more charge than its MSC25-7\% KB counterpart at higher current density (>50 $\left.\mathrm{mA} \mathrm{cm}^{-2}\right)$.

Figure 8 displays the specific capacitance of the two carbons that was calculated by dividing the inverse of the slopes (that is, capacitance) in the galvanostatic voltage profiles (Fig. 6) by the weight of carbon material loaded in the composite electrodes ( $c a .10 \mathrm{mg}$ ). The specific capacitance is invariant vs. current density within experimental error. The MSC25-7\% KB exhibits twice the capacitance ( $c a$. $200 \mathrm{~F} \mathrm{~g} \mathrm{~g}^{-1}$ ) of the NMC electrode ( $c a .120 \mathrm{~F} \mathrm{~g}^{-1}$ ), which certainly stems from the difference in surface area. The results shown in Fig. 7 and 8 indicate that the NMC electrode delivers a smaller specific capacitance than the other electrode. Based on the storable charge variation with charge-discharge rate, however, the NMC electrode outperforms the MSC25-7\% KB electrode at higher current.

A reason for the better rate capability of the NMC electrode can be found in the galvanostatic charge-discharge profiles shown in Fig. 6, where the voltage drop at the very early period of charging and discharging is visible. The voltage drop is larger in the MSC25$7 \% \mathrm{~KB}$ electrode than in the $\mathrm{NMC}$ electrode under the same current condition. Also, it becomes larger for both electrodes at higher current density. ${ }^{17}$ This ohmic voltage drop is certainly caused by the ESR in these cells. The more rapid decrease in the specific charging capacity of the MSC $25-7 \% \mathrm{~KB}$ with an increase in current density (Fig. 7) is caused by a larger ESR. As seen in Fig. 6, both the endof-charge $(0.0 \mathrm{~V})$ and end-of-discharge $(0.7 \mathrm{~V})$ are reached earlier due to the larger voltage drop at higher current density, thereby giving a smaller charge-discharge capacity. The results shown in Fig. 4 to 8 also reflect the relative ESR of the three electrodes. The MSC25 electrode has the largest ESR due to the largest $R_{\text {electrode }}$ (Table I). The addition of KB to MSC25 reduces the ESR by decreasing the $R_{\text {electrode }}$. It is interesting to note, however, that the MSC25-7\% KB electrode has a larger ESR than its NMC counterpart (Fig. 6) even if its $R_{\text {electrode }}$ is comparable to that of the NMC electrode (Table I). Note that the $R_{\text {bulk }}$ of the two electrodes are also comparable because the same cell configuration was used. It is thus very likely that $R_{\text {pore }}$ is the dominant factor affecting the overall ESR.

Measurement of ESR via RC time constants.-In order to estimate the ESR, the $R C$ time constants of two carbon electrodes were measured using chronoamperometry. ${ }^{9}$ For this measurement, a
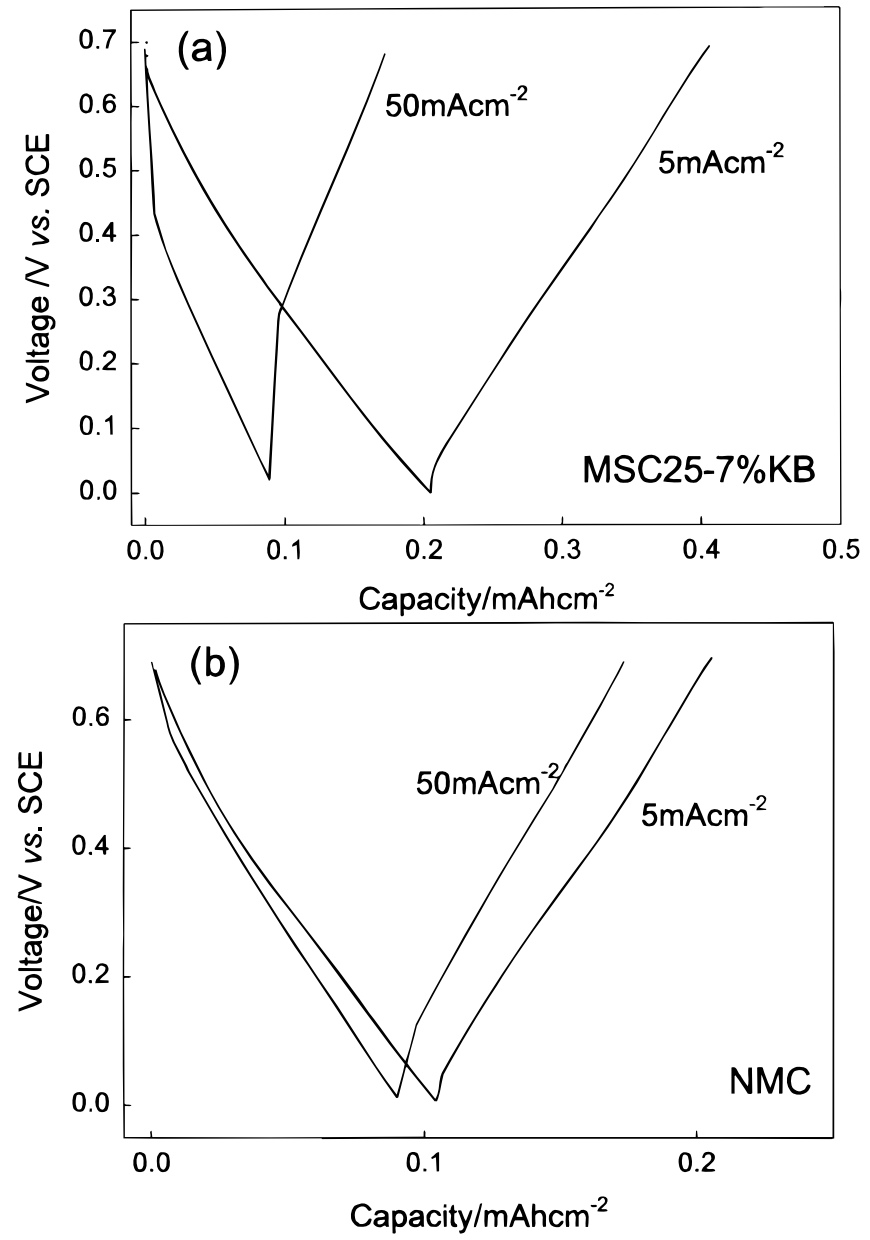

Figure 6. The galvanostatic charge-discharge voltage profiles recorded with two carbon composite electrodes at 5 and $50 \mathrm{~mA} \mathrm{~cm}^{-2}$. The inverse of the slope corresponds to the capacitance. Note the difference in the voltage drop between the two electrodes that reflects the relative ESR magnitude. 


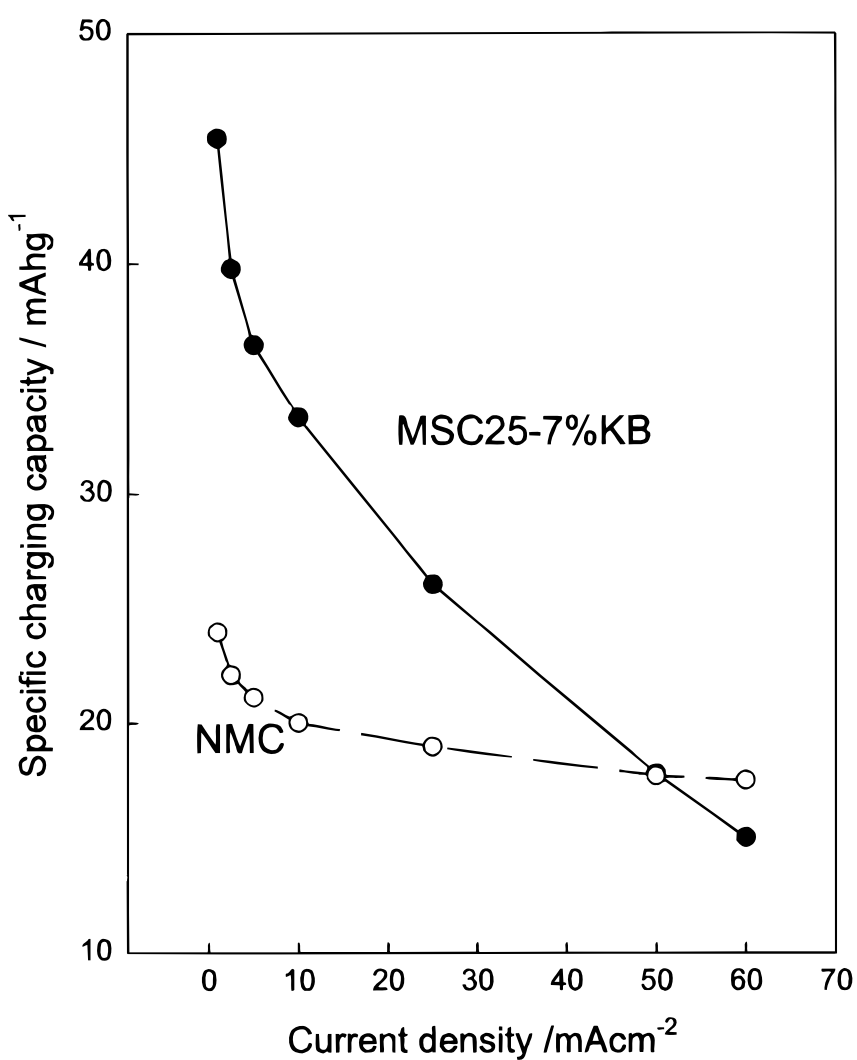

Figure 7. The specific charging capacity of two carbon electrodes as a function of current density. Note that the NMC electrode outperforms MSC25 at higher current density $\left(>50 \mathrm{~mA} \mathrm{~cm}^{-2}\right)$.

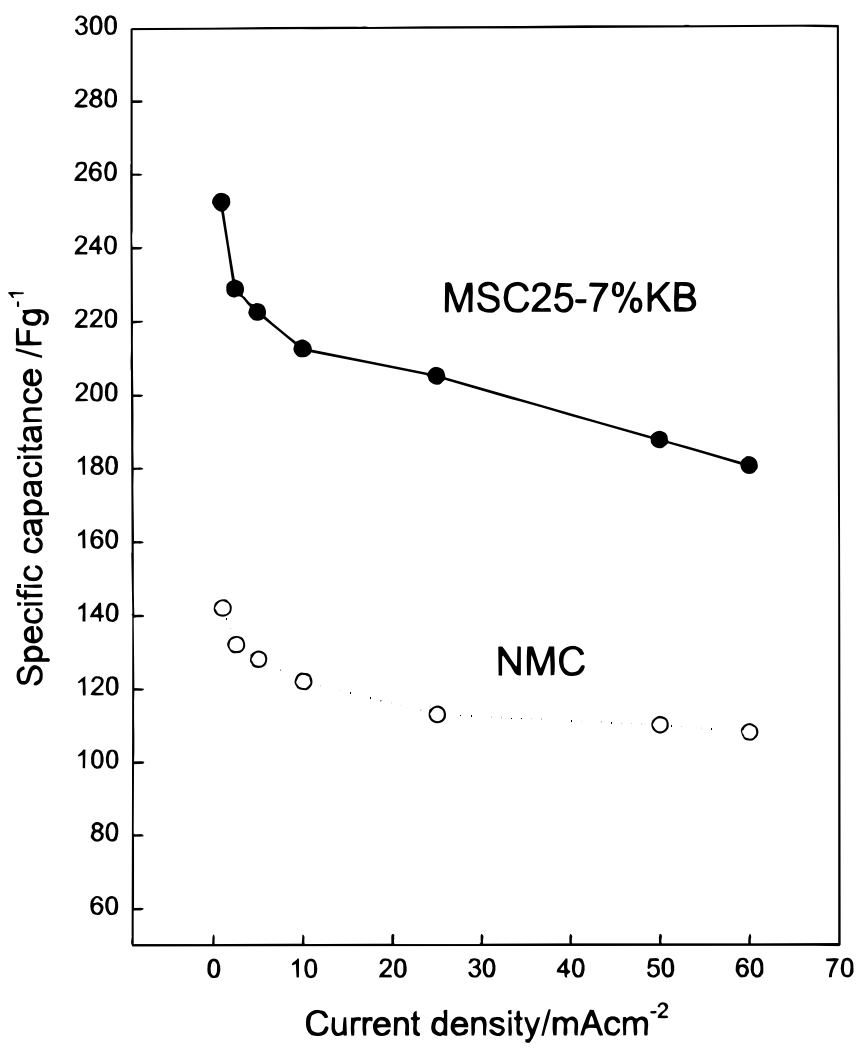

Figure 8. The specific capacitance obtained from galvanostatic charge-discharge voltage profiles for two carbon materials (some examples are given in Fig. 6). The inverse of the slope (capacitance) in Fig. 6 was divided by the weight of carbon material loaded in each electrode (ca. $10 \mathrm{mg}$ ).

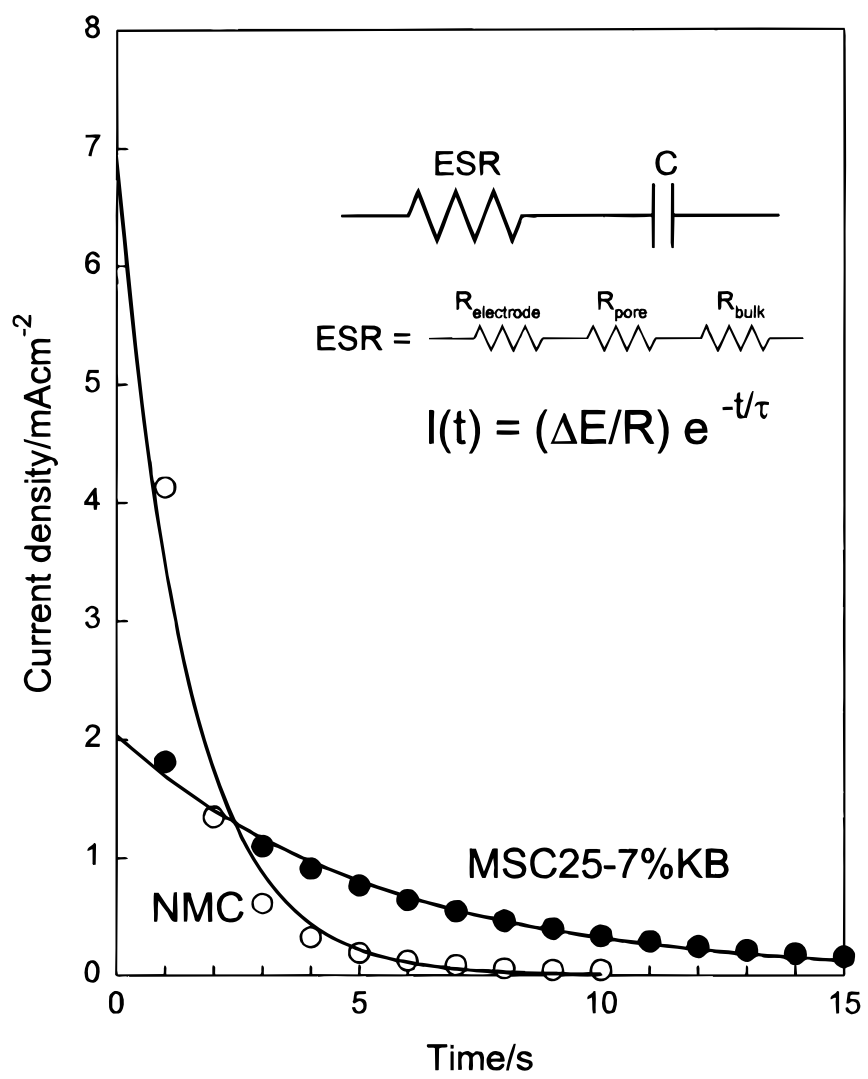

Figure 9. The typical current transient from a chronoamperometry experiment. A potential step $(10 \mathrm{mV})$ was applied from $0.2 \mathrm{~V}(\mathrm{vs}$. SCE). The equivalent circuit is depicted in the inset. The circles are the observed current transients and the solid lines represent the best fit with the equation indicated in the inset.

potential step $(10 \mathrm{mV})$ was applied from $0.2 \mathrm{~V}$, and the resulting current transients were recorded. This base potential $(0.2 \mathrm{~V})$ was selected because the pseudocapacitance arising from quinone-hydroquinone couples is absent in this potential range (see Fig. 4 or 5). The exponentially decaying current transients were fitted with the equation indicated in the inset of Fig. 9, from which the ESR and $C$ values were obtained. ${ }^{10}$ In Fig. 9, the circles represent the sampled currents, and the solid line corresponds to the fitted one. Table II lists the best-fit values of ESR, $C_{\text {obs }}$, and time constant $\left(\tau_{\text {obs }}\right)$ for two electrodes along with other electrochemical parameters. As seen, the MSC25-7\% KB electrode has larger values in both ESR and $C_{\text {obs }}$, thereby giving a larger $\tau_{\text {obs. }}$. From the $R_{\text {electrode }}$ and $R_{\text {bulk }}$ values that were measured separately, $R_{\text {pore }}$ was calculated. The MSC $25-7 \% \mathrm{~KB}$ electrode shows a 5.6 times larger $R_{\text {pore }}$ than the NMC electrode. Table II also compares the $C_{\text {obs }}$ values to those estimated from the plateau region in the capacitance $v s$. voltage profiles (Fig. 5). The ESRs were also compared to those estimated from the voltage drop in the galvanostatic charge-discharge profiles (Fig. 6). There appears a reasonable match between the parameters, demonstrating the accuracy of the measurements and the correctness of data interpretation.

The results listed in Table II indicate that the ESR in the MSC25$7 \% \mathrm{~KB}$ electrode is about 3.3 times larger than that in the NMC electrode, and that the dominant component in the ESR is $R_{\text {pore }}$. The larger $R_{\text {pore }}$ in the MSC25-7\% KB electrode likely arises from slower ionic motion in the randomly connected micropores. In the case of NMC, however, faster ionic motion is expected in the regularly interconnected mesopores, therefore the much smaller $R_{\text {pore }}$ is readily understood. The larger ESR in the MSC25-7\% KB accounts for the more pronounced voltage drop in Fig. 6 and also for the poorer rate capability shown in Fig. 7. The difference in the time constant of two electrodes also explains the results presented in Fig. 4 and 5. The more rectangular-shaped cyclic voltammograms and capaci- 
Table II. The ESR, capacitance $\left(C_{\text {obs }}\right)$ and time constant $\left(\tau_{\text {obs }}\right)$ obtained from a chronoamperometry experiment.

\begin{tabular}{lcl} 
& MSC25-7\%KB & NMC \\
\hline $\mathrm{ESR}\left(\Omega \mathrm{cm}^{2}\right)$ & 4.92 & 1.50 \\
$C_{\text {obs }}\left(\mathrm{F} \mathrm{cm}^{-2}\right)$ & 1.09 & 0.65 \\
$\tau_{\text {obs }}(\mathrm{s})$ & 5.36 & 0.98 \\
$R_{\text {electrode }}\left(\Omega \mathrm{cm}^{2} \mathrm{a}\right)$ & 0.02 & 0.02 \\
$R_{\text {bulk }}\left(\Omega \mathrm{cm}^{2 \mathrm{~b}}\right)$ & 0.73 & 0.73 \\
$R_{\text {pore }}\left(\Omega \mathrm{cm}^{2 \mathrm{c}}\right)$ & 4.17 & 0.75 \\
$\mathrm{R}\left(\Omega \mathrm{cm}^{2 \mathrm{~d}}\right)$ & 4.8 & 1.6 \\
$\mathrm{C}\left(\mathrm{F} \mathrm{cm}^{-2} \mathrm{e}\right)$ & 1.1 & 0.6
\end{tabular}

${ }^{a}$ The electrode resistance was calculated from the conductivity data (Table I); the apparent electrode area is $2 \mathrm{~cm}^{2}$ and the electrode thickness is $90 \mu \mathrm{m}$.

${ }^{\mathrm{b}}$ The bulk electrolyte resistance was calculated from the conductivity ( $0.68 \mathrm{~S} \mathrm{~cm}^{-1}$ for $2.0 \mathrm{M} \mathrm{H}_{2} \mathrm{SO}_{4}$ ); the apparent electrode area is $2 \mathrm{~cm}^{2}$ and the interelectrode gap is $0.5 \mathrm{~cm}$.

${ }^{\mathrm{c}}$ The electrolyte resistance in the pores was calculated by subtracting the $R_{\text {electrode }}$ and $R_{\text {bulk }}$ from the ESR.

${ }^{\mathrm{d}}$ The ESR estimated from the ohmic voltage drop in the galvanostatic charge-discharge curves recorded at $50 \mathrm{~mA} \mathrm{~cm}^{-2}$ (Fig. 6).

${ }^{\mathrm{e}}$ The capacitance estimated from the plateau value in the capacitance vs. voltage profiles shown in Fig. 5.

tance $v s$. voltage profiles are certainly the result of a smaller time constant for the NMC electrode.

The dominance of $R_{\text {pore }}$ in ESR and its importance in affecting overall EDLC performance is further demonstrated by providing the results obtained at lower electrolyte concentration. Figure 10 shows the specific charging capacity obtained from galvanostatic chargedischarge cycling at $50 \mathrm{~mA} \mathrm{~cm}{ }^{-2}$ with a variation in the electrolyte concentration. The bulk electrolyte conductivity of sulfuric acid was scaled in this figure. The charging capacity of the NMC electrode does not decrease much even with lower $\mathrm{H}_{2} \mathrm{SO}_{4}$ concentrations, whereas a substantial decrease is observed with the MSC25-7\% KB electrode. From the fact that the electrode resistances are comparable, and the bulk electrolyte conductivity changes with the same dependance, the concentration-dependent feature can be explained by the difference in the electrolyte conductivity in pores. That is, the electrolyte conductivity in randomly connected micropores decreases more significantly than in the regularly connected mesopores as the electrolyte becomes more diluted.

\section{Conclusion}

1. The NMC has an well-ordered pore structure with uniform mesopores of $2.3 \mathrm{~nm}$. In the case of MSC25, however, the random cage-like micropores of $<2.0 \mathrm{~nm}$ are dominant. The surface area of NMC and MSC25 was estimated to be 1257 and $1970 \mathrm{~m}^{2} \mathrm{~g}^{-1}$, respectively. In proportion to the surface area, the NMC electrode exhbits a smaller specific capacitance $\left(c a .120 \mathrm{~F} \mathrm{~g}^{-1}\right)$ than the MSC25 electrode ( $\left.c a .200 \mathrm{~F} \mathrm{~g}^{-1}\right)$.

2. The NMC electrode shows a higher critical scan rate than the MSC $25-7 \% \mathrm{~KB}$ due to a smaller $R C$ time constant, which is evidenced by both the more rectangular shaped cyclic voltammograms and capacitance $v s$. voltage profiles. The NMC electrode exhibits 1.8 times smaller capacitance and 3.3 times smaller ESR as compared to those observed with its MSC25-7\%KB counterpart.

3. The storable charge in the NMC electrode estimated from galvanostatic charge-discharge cycling amounts to about $20 \mathrm{mAh} \mathrm{g}^{-1}$ regardless of the imposed current density. The MSC25-7\% KB electrode stores more charges than the NMC electrode at lower current density, but its storable charge decreases rapidly with an increase in current density. Consequently, the NMC electrode stores more charges than the MSC25-7\% KB electrode at a high current.

4. The smaller $R C$ time constant and better rate capability of NMC likely results from the lower equivalent series resistance,

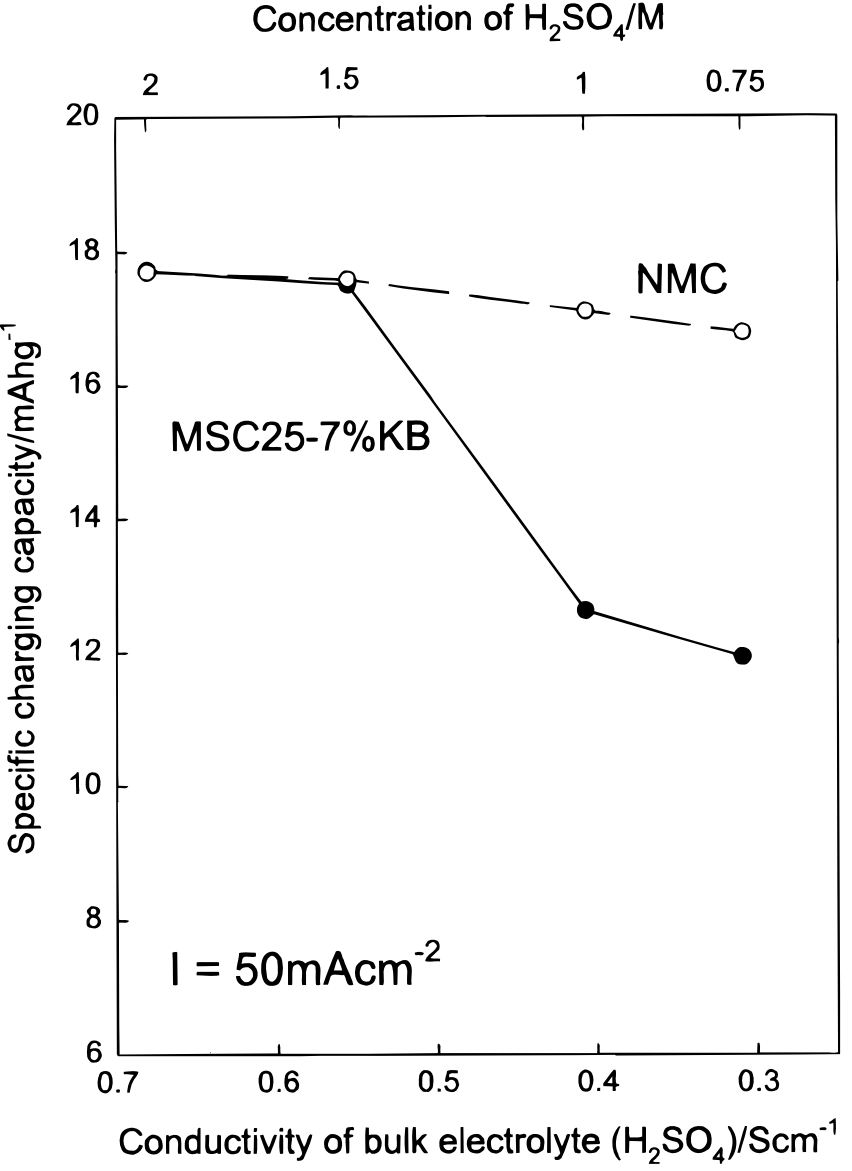

Figure 10. Change of the specific charging capacity with $\mathrm{H}_{2} \mathrm{SO}_{4}$ concentration and bulk electrolyte conductivity. The data were taken from galvanostatic charge-discharge cycling at $50 \mathrm{~mA} \mathrm{~cm}^{-2}$.

which is caused mainly by a lower electrolyte resistance in its pores. The presence of regularly connected mesopores in NMC accounts for this feature.

This work was supported by the Brain Korea 21 Project.

\section{References}

1. K. Kinoshita and X. Chu, in Electrochemical Capacitors, F. M. Delnick and M. Tomkiewicz, Editors, PV 95-29, p. 171, The Electrochemical Society Proceedings Series, Pennington, NJ (1996).

2. B. McEnaney and T. J. Mays, in Introduction to Carbon Science, H. Marsh, Editor p. 156, Butterworths, London (1989).

3. J. F. Byrne and H. Marsh, in Porosity in Carbons, J. W. Patrick, Editor, pp. 12-14, Edward Arnold, London (1995).

4. J. Lee, S. Yoon, T. Hyeon, S. M. Oh, and K. B. Kim, Chem. Commun., 21, 2177 (1999).

5. J. Lee, S. Yoon, S. M. Oh, C.-H. Shin, and T. Hyeon, Adv. Mater., 12, 359(2000)

6. A. N. Wennerberg and T. M. O'Grady, U.S. Pat. 4,082,694 (1978).

7. L. J. van der Pauw, Philips Tech. Rev., 20, 220 (1958/1959).

8. A. Espinola, P. M. Miguel, M. R. Salles, and A. R. Pinto, Carbon, 24, 337 (1986).

9. M. G. Sullivan, M. Bartsch, R. Kotz, and O. Haas, in Electrochemical Capacitors II, F. M. Delnick, D. Ingersoll, X. Andrieu, and K. Naoi, Editors, PV 96-25, p. 192, The Electrochemical Society Proceedings Series, Pennington, NJ (1997).

10. A. J. Bard and L. R. Faulkner, Electrochemical Methods: Fundamentals and Applications, pp.11-15, John Wiley \& Sons, New York (1980).

11. T. Zheng, Q. Zhong, and J. R. Dahn, J. Electrochem, Soc., 142, L211 (1995).

12. C. Wu and T. Bein, Science, 266, 1013 (1994).

13. L. G. Austin and E. G. Gagnon, J. Electrochem. Soc., 120, 251 (1973).

14. E. G. Gagnon, J. Electrochem. Soc., 120, 1052 (1973).

15. K. Kinoshita, Carbon: Electrochemical and Physicochemical Properties, p. 299, John Wiley \& Sons, New York (1988)

16. M. Muller and B. Kastening, J. Electroanal. Chem., 374, 149 (1994).

17. M. Yaniv and A. Soffer, J. Electrochem. Soc., 123, 506 (1973). 